\title{
A Comparative Assessment of Spalart-Shur Rotation/Curvature Correction in RANS Simulations in a Centrifugal Pump Impeller
}

\author{
Ran Tao, Ruofu Xiao, Wei Yang, and Fujun Wang \\ College of Water Resources and Civil Engineering, China Agricultural University, Beijing 100083, China \\ Correspondence should be addressed to Ruofu Xiao; xrf@cau.edu.cn
}

Received 10 June 2014; Accepted 29 August 2014; Published 30 September 2014

Academic Editor: Shaofan Li

Copyright ( 2014 Ran Tao et al. This is an open access article distributed under the Creative Commons Attribution License, which permits unrestricted use, distribution, and reproduction in any medium, provided the original work is properly cited.

\begin{abstract}
RANS simulation is widely used in the flow prediction of centrifugal pumps. Influenced by impeller rotation and streamline curvature, the eddy viscosity models with turbulence isotropy assumption are not accurate enough. In this study, Spalart-Shur rotation/curvature correction was applied on the SST $k-\omega$ turbulence model. The comparative assessment of the correction was proceeded in the simulations of a centrifugal pump impeller. CFD results were compared with existing PIV and LDV data under the design and low flow rate off-design conditions. Results show the improvements of the simulation especially in the situation that turbulence strongly produced due to undesirable flow structures. Under the design condition, more reasonable turbulence kinetic energy contour was captured after correction. Under the low flow rate off-design condition, the prediction of turbulence kinetic energy and velocity distributions became much more accurate when using the corrected model. So, the rotation/curvature correction was proved effective in this study. And, it is also proved acceptable and recommended to use in the engineering simulations of centrifugal pump impellers.
\end{abstract}

\section{Introduction}

Reynolds-averaged Navier-Stokes (RANS) simulation provides effective solutions for numerical simulations in engineering. By solving the time-averaged N-S equations, RANS simulation reduces the consumption of computing resources on the premise of keeping sufficient accuracy. Based on the Boussinesq assumption $[1,2]$, eddy viscosity models (EVM) directly established the relationship between Reynolds stress tensor and the traceless mean strain rate tensor. Because of the simplicity and stability, EVMs are widely used in engineering simulations. However, because of the coordinate invariance, EVMs are insensitive to streamline curvature and system rotation [3]. Moreover, with the turbulence isotropy assumption, the rotation effect on turbulent flow is difficult to describe. However, under the influences of the nonlinear wave induced by the Coriolis force, the flow structure will change [4]. So, a proper correction for EVM is necessary in the RANS simulation of rotating and swirling flow.

To describe the effects of rotation or streamline curvature on a turbulent flow, Bradshaw [5] first proposed the gradient
Richardson number Ri. Combined with the low-Reynoldsnumber $k-\varepsilon$ model, Khodak and Hirsch [6] introduced a new form of $R i$ number which allows including the influence of curvature and rotation on the three-dimensional turbulent flow. It improved the prediction accuracy of mean velocity and Reynolds stresses in verification cases. On the basis of Galilean invariance, Spalart and Shur [3] differentiated the property between streamline curvature and system rotation and also introduced a new Ri number. By establishing intermediate variables, this new Ri number was used to correct the turbulence production term of EVM. This correction method was applied to Spalart-Allmaras model by Shur et al. [7] and proved to be much more accurate than the original S-A model. Moreover, Dufour et al. $[8,9]$ corrected the $k-\varepsilon$ model and compared it with the Spalart-Shur corrected S-A model through a compressor case. Improvements were obtained after the rotation and curvature correction for the corrected $k-\varepsilon$ model. Smirnov and Menter [10] also applied the SpalartShur correction to SST $k-\omega$ model that is known as the SST-RC model. The computational accuracy was proved to be significantly improved with just a little increase of the 
time costs. Then, Dhakal and Walters [11] corrected the SST $k-\omega$ model based on the correction method by York et al. [12]. The new model was compared with the SST-RC model and was proved accurate without compromising stability and efficiency. With the modified mean-flow time scale, Hellsten [13] also introduced a new $R i$ number to correct the $\omega$ equation of SST $k-\omega$ model. This new corrected model, which is called SST-RC-Hellsten model, was sensitized on the effects of system rotation and streamline curvature with slight improvement on the numerical behavior. Based on SpalartShur correction method and Hellsten's time scale, Zhang and Yang [14] corrected the S-A model and got reasonable results in a U-turn duct case.

In general, all the correction methods mentioned above were theoretically feasible for swirling turbulent flow. For centrifugal pumps, the internal flow regime varies with the operating condition [15]. Under off-design conditions, the turbulent flow in the impeller is much more complicated due to the vortex, backflow, and other secondary flow structures. Hence, it is necessary to ensure the simulation accuracy of centrifugal pumps under the influence of system rotation and streamline curvature. However, in the vast majority of RANS simulations of centrifugal pumps, the rotation and curvature effects are not considered when using EVM. In order to evaluate the Spalart-Shur rotation/curvature correction in the centrifugal pump cases, RANS simulations were conducted with both the original and the corrected SST $k-\omega$ models. The simulation results were compared with the experimental data by particle image velocimetry and laser Doppler velocimetry $[16,17]$.

\section{Turbulence Modeling}

As mentioned above, the SST-RC turbulence model [10] was used in the turbulent flow simulation. Compared with the original SST $k-\omega$ model [18], in consideration of the turbulence anisotropy, the rotation/curvature correction coefficient $f_{r 1}$ was introduced as a multiplier of the turbulence production term $P$ in the turbulence kinetic energy $k$ equation and specific dissipation rate $\omega$ equation. So, the production term $P$ is defined as follows:

$$
P=f_{r 1} \tau_{i j} \frac{\partial u_{i}}{\partial x_{j}},
$$

where $f_{r 1}$ is given empirically with specific limiters as follows:

$$
\begin{aligned}
f_{r 1} & =\max \left[\min \left(f_{\text {rot }}, 1.25\right), 0\right] \\
f_{\text {rot }} & =\left(1+C_{r 1}\right) \frac{2 r^{*}}{1+r^{*}}\left[1-C_{r 3} \tan ^{-1}\left(C_{r 2} \widehat{r}\right)\right]-C_{r 1},
\end{aligned}
$$

where $C_{r 1}, C_{r 2}$, and $C_{r 3}$ are constants valued as 1.0, 2.0, and 1.0 respectively. The remaining functions are defined as follows:

$$
\begin{aligned}
r^{*} & =\frac{S}{W}, \\
\widehat{r} & =\frac{2 W_{i k} S_{j k}}{W D^{3}}\left[\frac{\mathrm{D} S_{i j}}{\mathrm{D}_{t}}+\left(\varepsilon_{i m n} S_{j n}+\varepsilon_{j m n} S_{i n}\right) \Omega_{m}^{\mathrm{rot}}\right],
\end{aligned}
$$

$$
\begin{aligned}
S^{2} & =2 S_{i j} S_{i j}, \\
W^{2} & =2 W_{i j} W_{i j}, \\
D^{2} & =\max \left(S^{2}, 0.09 \omega^{2}\right),
\end{aligned}
$$

where $\Omega^{\text {rot }}$ is the rotation rate of the reference frame and the term $D S_{i j} / D_{t}$ represents the Lagrangian derivative of the strain rate tensor. $S_{i j}$ is the strain rate tensor and $W_{i j}$ is the rotation rate tensor by

$$
\begin{aligned}
S_{i j} & =\frac{1}{2}\left(\frac{\partial u_{i}}{\partial x_{j}}+\frac{\partial u_{j}}{\partial x_{i}}\right) \\
W_{i j} & =\frac{1}{2}\left(\frac{\partial u_{i}}{\partial x_{j}}+\frac{\partial u_{j}}{\partial x_{i}}\right)+\varepsilon_{m j i} \Omega_{m}^{\text {rot }} .
\end{aligned}
$$

The $\tau_{i j}$ in (1) is the Reynolds stress tensor. In the Boussinesq assumption [2], $\tau_{i j}$ is proportional to the strain rate tensor $S_{i j}$ by

$$
\tau_{i j}=\mu_{t}\left(2 S_{i j}-\frac{2}{3} \frac{\partial u_{k}}{\partial x_{k}} \delta_{i j}\right),
$$

where $\mu_{t}$ is the turbulent eddy viscosity. By applying the rotation/curvature correction, anisotropic effects were considered in the simulation when solving the turbulence equations.

\section{Numerical Simulation}

To assess the Spalart-Shur rotation/curvature correction in RANS simulation of centrifugal pump, the internal flow in the impeller was numerically simulated. Based on the numerical results and available experimental data [16], the flow details were comparatively investigated under both the design and low flow rate off-design conditions.

3.1. Pump Impeller Model. The scheme of the investigated centrifugal pump impeller is shown in Figure 1. The specificspeed $N_{s}$ of this impeller is about 26.3 calculated by

$$
N_{s}=3.65 \frac{n \sqrt{Q}}{H^{3 / 4}},
$$

where $n$ is the rotational speed of $725 \mathrm{r} / \mathrm{min}, Q_{d}$ is the design flow rate of $3.06 \times 10^{-3} \mathrm{~m}^{3} / \mathrm{s}$, and $H_{d}$ is the design head of $1.75 \mathrm{~m}$. The geometrical parameters of impeller are shown in Table 1 and illustrated in Figure 1. Two operating conditions including $Q=1.0 Q_{d}$ and $Q=0.25 Q_{d}$ were simulated and performed in this study.

3.2. Flow Domain Discretization. The flow domain consisted of the impeller only. For a better geometric adaptability, tetrahedral mesh elements were used to discretize the impeller domain. For the usage of wall functions, $y^{+}$of the first element outside the walls should be set in the log-layer. So, prism boundary layers were used in the near wall region. 

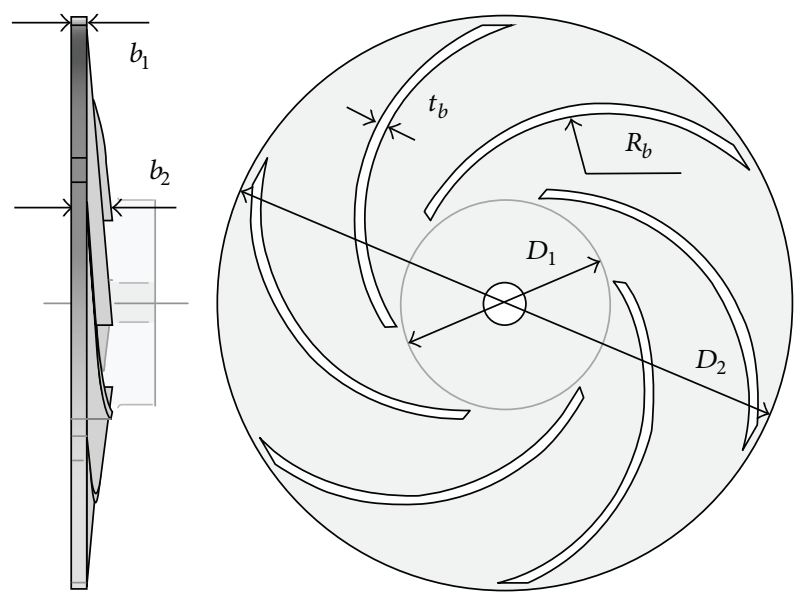

FIGURE 1: Scheme of the investigated centrifugal pump impeller.

TABLE 1: Geometrical parameters of impeller.

\begin{tabular}{lc}
\hline Parameter & Value \\
\hline Inlet diameter $D_{1}$ & $71.0 \mathrm{~mm}$ \\
Outlet diameter $D_{2}$ & $190.0 \mathrm{~mm}$ \\
Inlet height $b_{1}$ & $13.8 \mathrm{~mm}$ \\
Outlet height $b_{2}$ & $5.8 \mathrm{~mm}$ \\
Number of blades $Z$ & 6 \\
Blade thickness $t_{b}$ & $3.0 \mathrm{~mm}$ \\
Inlet blade angle $\beta_{1}$ & 19.7 degrees \\
Outlet blade angle $\beta_{2}$ & 18.4 degrees \\
Blade curvature radius $R_{b}$ & $70.0 \mathrm{~mm}$ \\
\hline
\end{tabular}

TABle 2: Detailed parameters of mesh scheme.

\begin{tabular}{lc}
\hline Parameter & Value \\
\hline Nodes & 406012 \\
Elements & 2046670 \\
Prism boundary layers & 5 \\
The first layer height & $0.1 \mathrm{~mm}$ \\
Boundary layer growth rate & 1.2 \\
\hline
\end{tabular}

Then, a mesh-size independence check was conducted to compromise the accuracy and costs in the simulation. By modifying the mesh size, the residuals of head and hydraulic efficiency was ensured to be less than $1 \times 10^{-3}$. By modifying the boundary layer height, the $y^{+}$values were controlled within the range from 1.53 to 26.97 so that the near-wall region could be solved with wall functions. The final mesh scheme is shown in Table 2 and Figure 2.

3.3. Simulation Settings. In this study, transient numerical simulations were conducted. Three dimensional incompressible N-S equations were solved in the simulation process. The fluid medium was set as water at 25 degree centigrade (density $\rho=997 \mathrm{~kg} / \mathrm{m}^{3}$ and dynamic viscosity $\mu=8.899 \times$ $\left.10^{-4} \mathrm{~kg} / \mathrm{m} \cdot \mathrm{s}\right)$. The reference frame was set as rotational with the speed of $725 \mathrm{r} / \mathrm{min}$. The reference pressure was $1 \mathrm{Atm}$. Mass flow inlet was set at the impeller inflow with the

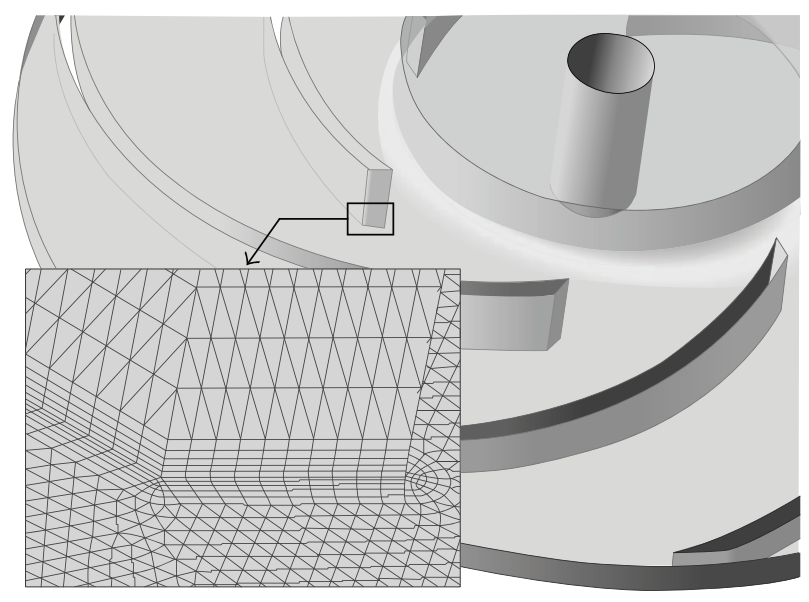

Figure 2: Schematic map of the mesh.

velocity normal to the boundary. Pressure outlet was set at the impeller outflow with a value of $0 \mathrm{~Pa}$. No-slip wall condition was given on the solid wall boundaries including hub, shroud and blades.

\section{Results and Discussion}

In order to fully assess the impacts of Spalart-Shur correction, as mentioned above, the simulations were proceeded under the design $\left(1.0 Q_{d}\right)$ and off-design $\left(0.25 Q_{d}\right)$ conditions. The design condition is the most important operating condition of pump. A correct simulation of the flow details is obviously significant. The off-design condition is also crucial. Undesirable flow structures make the flow hard to predict. For this reason, the improvements of simulation accuracy are necessary. Hence, based on the CFD results, the comparative assessment of the rotation/curvature correction and discussions are given as follows.

4.1. Flow under the Design Condition. Under the design condition $\left(1.0 Q_{d}\right)$, the velocity field on the spanwise $50 \%$ surface was simulated and compared with the LDV data [16] as shown in Figure 3. It can be seen that the flow regime is smooth, stable, and uniform among all the impeller passages.

The correction coefficient $f_{r}$ contour on the spanwise $50 \%$ surface is shown in Figure 4. It indicates the enhancement or reduction of local turbulence production under the influence of rotation and curvature. As the multiplier of turbulence production term, the value of $f_{r}$ was almost 1.0 in the vast majority of impeller domain. However, small scale of reduction was detected at the blade leading edge (LE) and in the near suction surface (SS) region.

Figure 5 shows the comparison map of turbulence kinetic energy $k_{2 D}$. In the impeller, fluid separated from the blade surface while flowing around the LE. High $k_{2 D}$ region occurred due to the small scale local separation. Also, in the near SS region, high turbulence occurred due to the local unattached flow. After the rotation/curvature correction, the range of high $k_{2 D}$ region became smaller than before because 


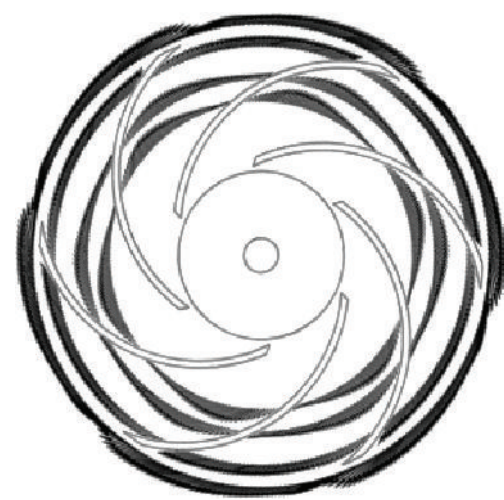

(a) $\operatorname{SST} k-\omega$

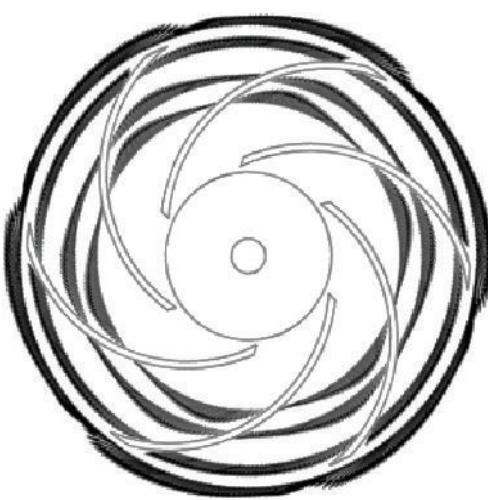

(b) SST-RC

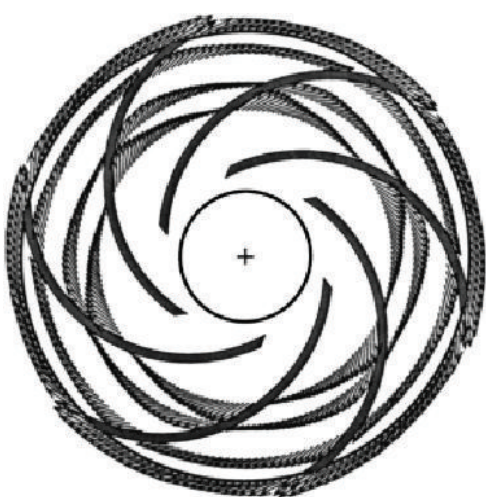

(c) $\mathrm{LDV}$

FIgURE 3: Velocity field on spanwise $50 \%$ surface under $1.0 Q_{d}$.

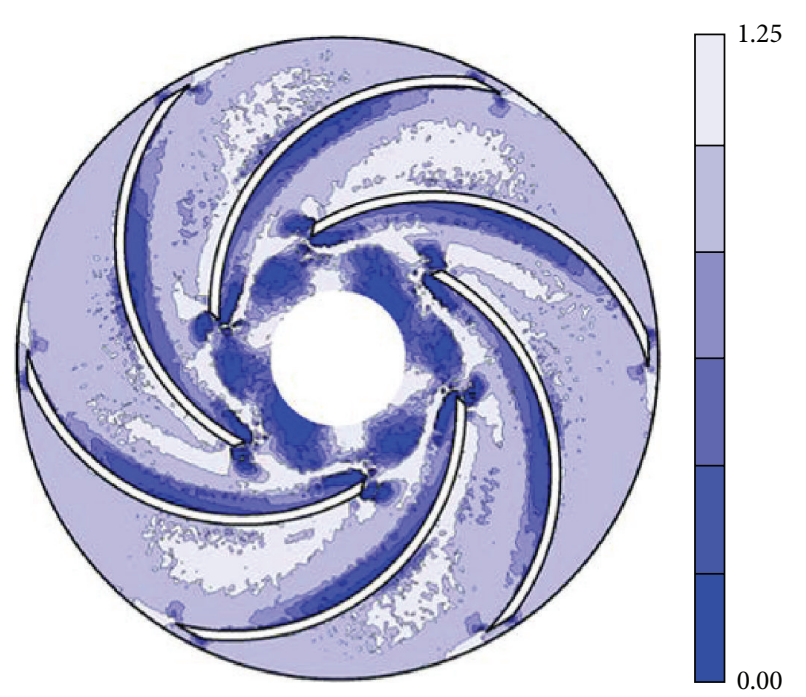

Figure 4: Correction coefficient $f_{r}$ on spanwise $50 \%$ surface at $1.0 Q_{d}$.

of the turbulence reduction. As shown in Figure 5, by comparing the $k_{2 D}$ value and pattern, the result by SST-RC model was more consistent with the PIV data [16].

Additionally, the relative velocity distributions on the spanwise $50 \%$ surface are analyzed and shown as follows. The radial component $\left(W_{r}\right)$ and tangential component $\left(W_{t}\right)$ in three different radius' positions were plotted, respectively, in Figure $6\left(R_{2}=0.5 D_{2}\right)$. Considering the flow uniformity among all the passages, the velocity distributions were compared based on just a single passage. Seen from the velocity curves, the velocities magnitude by SST-RC model was just slightly bigger than that by SST $k$ - $\omega$ model. In consideration of the deviations between the PIV and LDV data [16], both the original and the corrected turbulence models get the similar velocity distribution regularities.

In general, the impact of rotation/curvature correction on the simulation accuracy was not obvious under $1.0 Q_{d}$. Nevertheless, there was no obvious extra time cost when using SST-RC model instead of SST $k-\omega$ model. So, it would be reasonable to use the SST-RC model in the simulation of centrifugal pump under the design condition.

4.2. Flow under the Low Flow Rate Off-Design Condition. Under the low flow rate off-design condition $\left(0.25 Q_{d}\right)$, the velocity field on the spanwise $50 \%$ surface was also simulated and compared with the LDV data [16] as shown in Figure 7. With the flow rate decreasing, flow regime in the impeller became undesirable with secondary flow structures. As shown in the velocity field map, fluid did not flow along the direction of blade geometry. Back flow from outlet to inlet occurred in the passage. Lateral secondary flow from blade pressure surface (PS) to suction surface (SS) also occurred. Under the influence of all the disordered flow structures, the flow uniformity among all the passages disappeared. Some passages were blocked by secondary flow, but some other passages were smoother. As shown in the LDV experiment, the blocked passage and unblocked passage occurred alternately in the impeller [16]. This phenomenon was also captured by numerical simulations. In this situation, the streamline curvature under $0.25 Q_{d}$ became more obvious than that under $1.0 Q_{d}$.

The correction details under $0.25 Q_{d}$ are shown in Figure 8. As plotted in the $f_{r}$ contour, reductions of turbulence production were detected in the vast majority of impeller domain. But influenced by the flow regime, the rotation/curvature correction was different in each passage. The passages marked " $\mathrm{A}$ " and " $\mathrm{B}$ " in Figure 8 represented the unblocked and blocked passages, respectively. In passage-A, the low $f_{r}$ region occurred at the whole blade SS. The high $f_{r}$ region occurred at the PS near LE and the midpassage near trailing edge (TE). In passage- $\mathrm{B}$, the low $f_{r}$ region occurred at SS near LE and midpassage near TE. The high $f_{r}$ region occurred at SS near TE.

Influenced by the differences of coefficient $f_{r}$, the turbulence kinetic energy $k_{2 D}$ had also changed after the correction. Figure 9 shows the $k_{2 D}$ contour on the spanwise $50 \%$ surface under $0.25 Q_{d}$. As shown in the contours, the turbulence kinetic energy was low in the unblocked " $\mathrm{A}$ " passages and was high in the blocked "B" passages. Due to the flow separation at LE and backflow at TE, the high $k_{2 D}$ region 


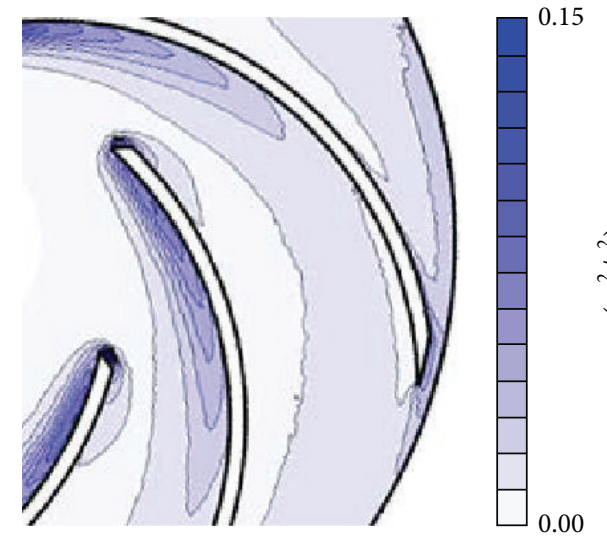

(a) $\operatorname{SST} k-\omega$

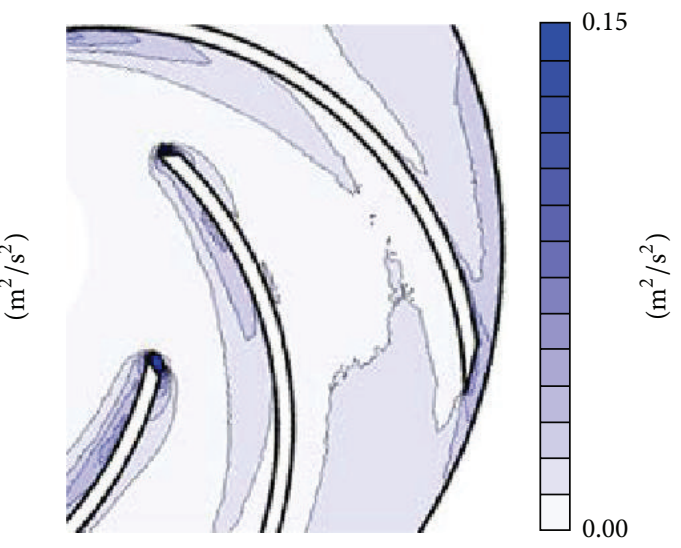

(b) SST-RC

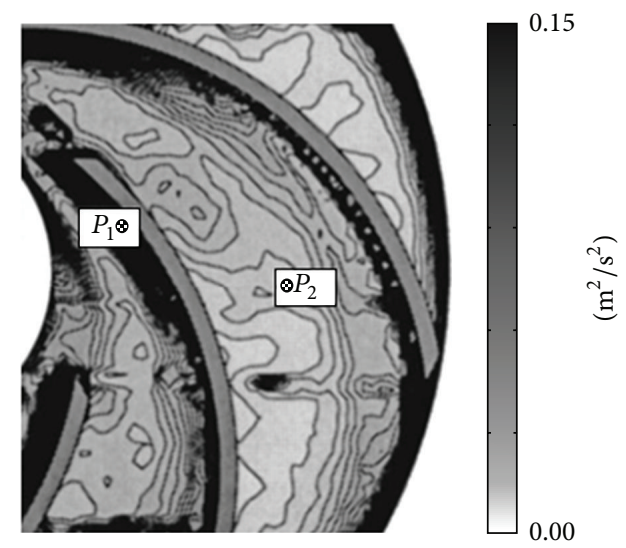

(c) PIV

FIGURE 5: Contour of turbulence kinetic energy $k_{2 D}$ on the spanwise $50 \%$ surface under $1.0 Q_{d}$.

occurred at LE and TE in "B" passages. On the contrary, because of the well-behaved flow regime, the $k_{2 D}$ value was lower in "A" passages. Compared with the PIV data [16], the location and intensity of $k_{2 D}$ were more reasonable after correction. Moreover, the alternately blocked phenomena were more obvious by using the SST-RC model.

To assess in details, the relative velocity distributions on the spanwise $50 \%$ surface are also plotted in Figure 10. Considering the differences of flow regime among all the passages, two adjacent passages ("A" and "B") were analyzed. The distributions of velocity components changed after correction. Compared with the experimental data [16], it can be seen that the results became more accurate. In particular, flow severely separated at blade LE with a stronger streamline curvature and higher turbulence. So, corrected by reducing the production of turbulence, radial and tangential velocities became more consistent with experiments especially at $0.5 R_{2}$.

All in all, the correction impact was obvious under $0.25 Q_{d}$. So, it is strongly recommended to use the corrected model under the low flow rate off-design condition.

\section{Conclusions}

By comparatively assessing the Spalart-Shur correction in the RANS simulations in centrifugal pump impeller under different operating conditions, conclusions can be drawn as follows.

(1) In the RANS simulations, the isotropous description of turbulence model is not perfect enough. The flow in a centrifugal pump impeller is strongly affected by the system rotation and streamline curvature. With the pump rotation, separation flow occurred at blade leading edge. Under different operating conditions, the scale of separation is also different. Particularly under low flow rate off-design conditions, fluid does not flow along the blade geometry; secondary flow structures become more and more obvious and occurred everywhere in the pump impeller passages.

(2) By the supplements of descriptions of turbulence anisotropy, turbulence production term is corrected. Verified by comparing the CFD results with experimental data, improvements are found after correction. Under the design condition, the impact of correction is not obvious but theoretically reasonable. Under the low flow rate off-design condition, simulation accuracy is significantly improved especially in the strong separation region. Moreover, there is no obvious extra time cost when using the corrected model. Hence, in the RANS simulations of centrifugal 

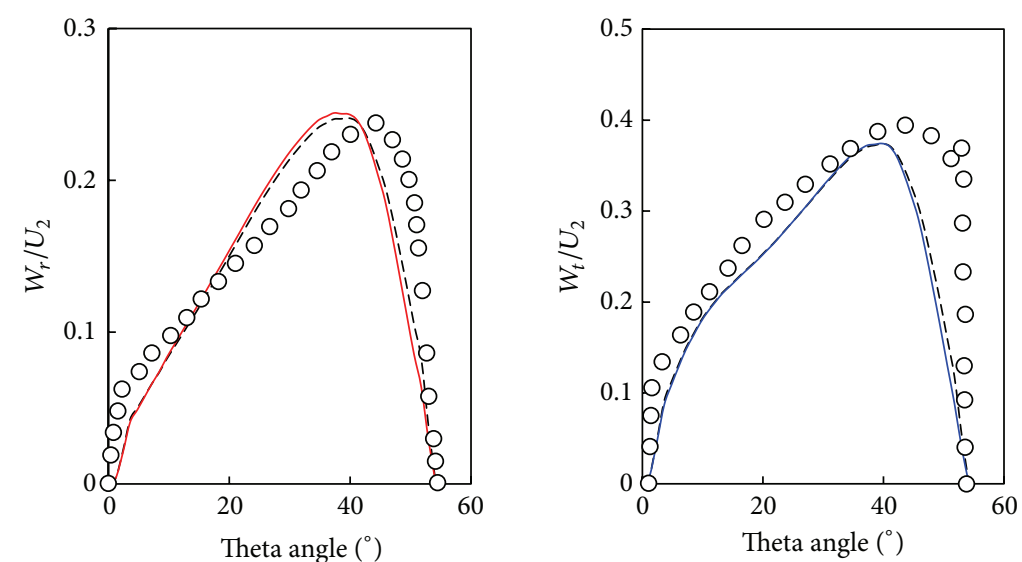

(a) Radius $R=0.50 R_{2}$
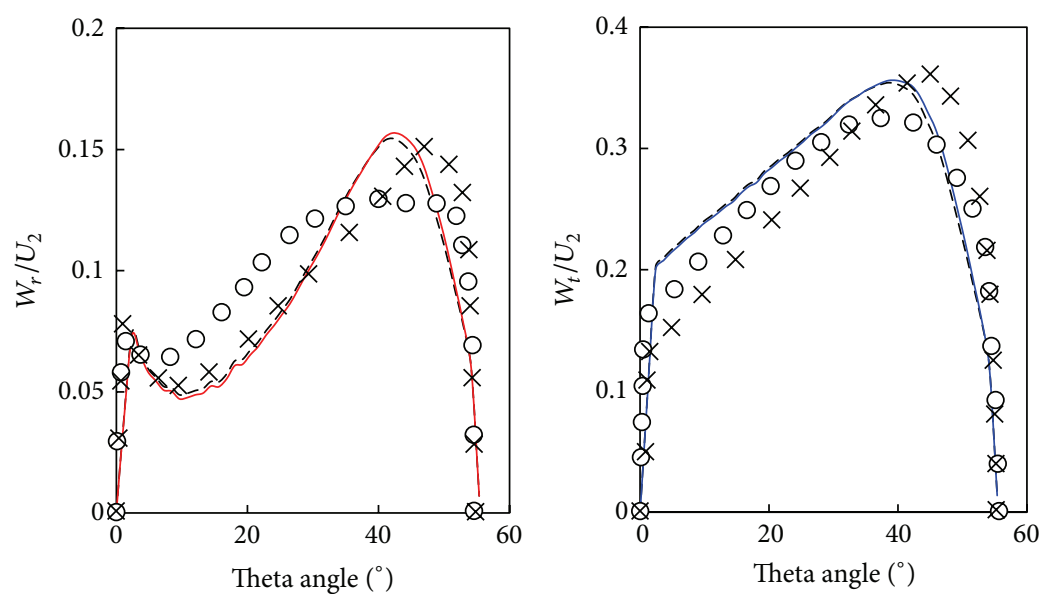

(b) Radius $R=0.75 R_{2}$
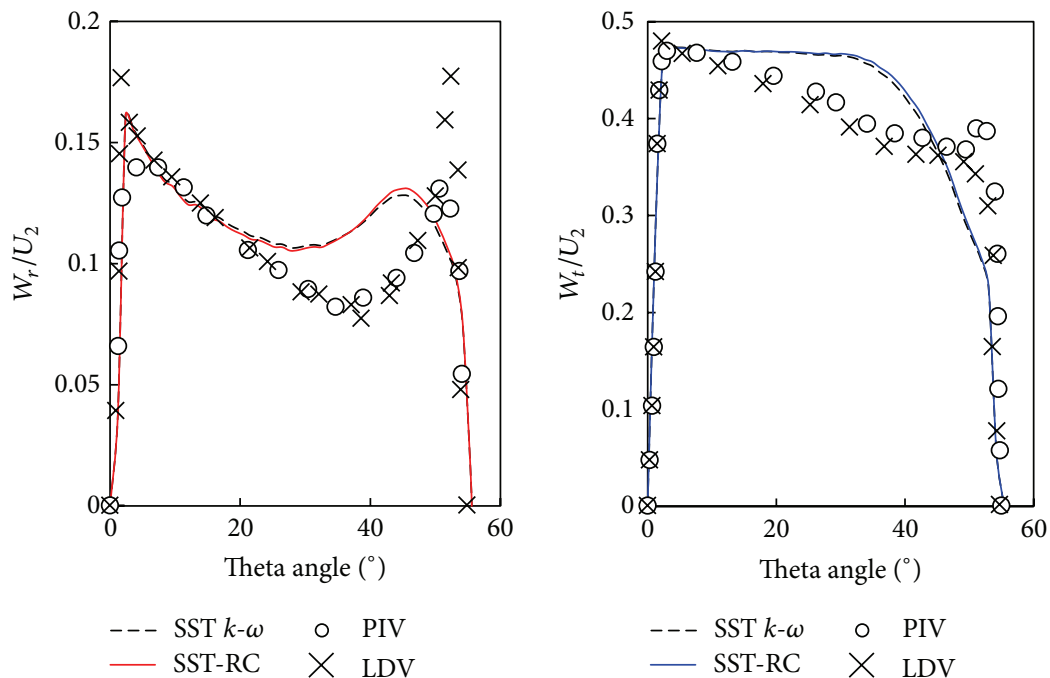

(c) Radius $R=0.98 R_{2}$

FIGURE 6: Relative velocity distributions on the spanwise $50 \%$ surface at radius of $0.50,0.75$, and $0.98 R_{2}$ under $1.0 Q_{d}$. 


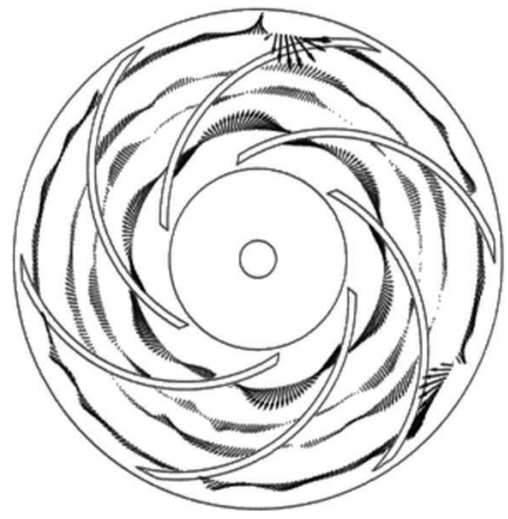

(a) SST $k$ - $\omega$

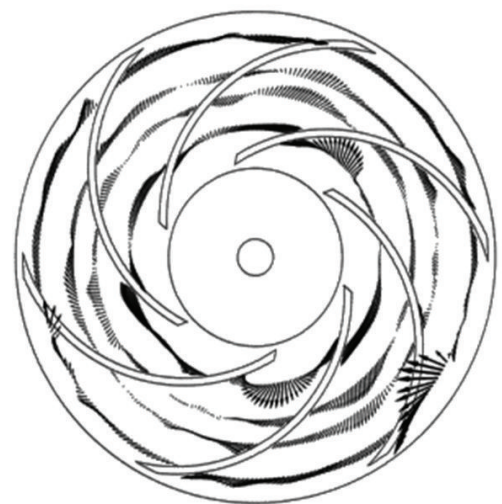

(b) SST-RC

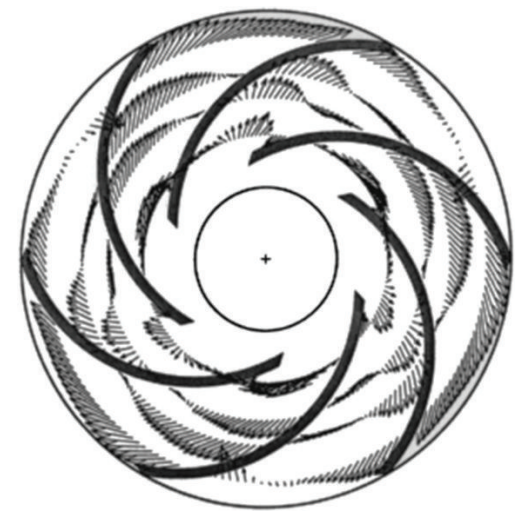

(c) LDV

FIGURE 7: Velocity field on spanwise $50 \%$ surface under $0.25 Q_{d}$.

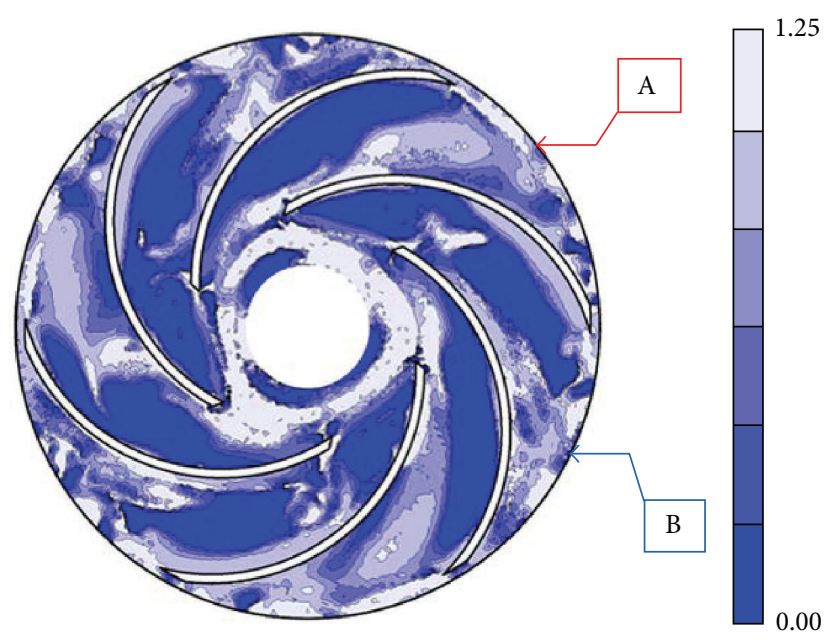

Figure 8: Correction coefficient $f_{r}$ on spanwise $50 \%$ surface at $0.25 Q_{d}$.

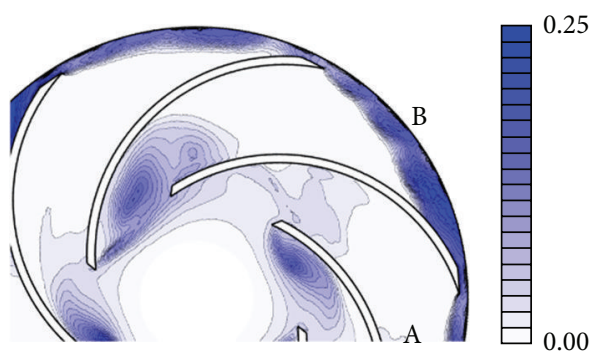

(a) SST $k$ - $\omega$

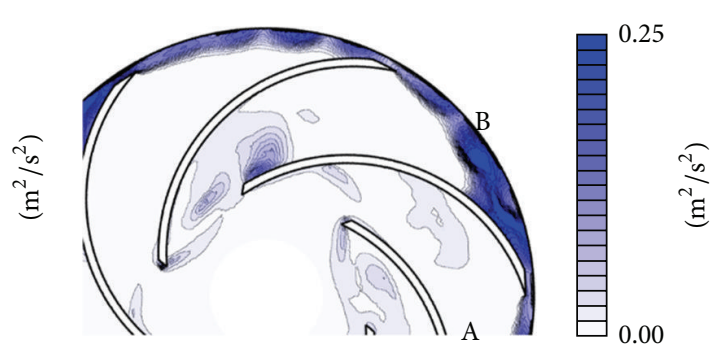

(b) SST-RC

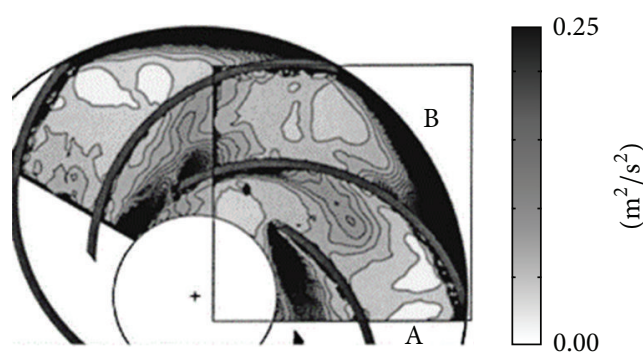

(c) PIV

FIgURE 9: The contour of turbulence kinetic energy $k_{2 D}$ on the spanwise $50 \%$ surface under $0.25 Q_{d}$. 

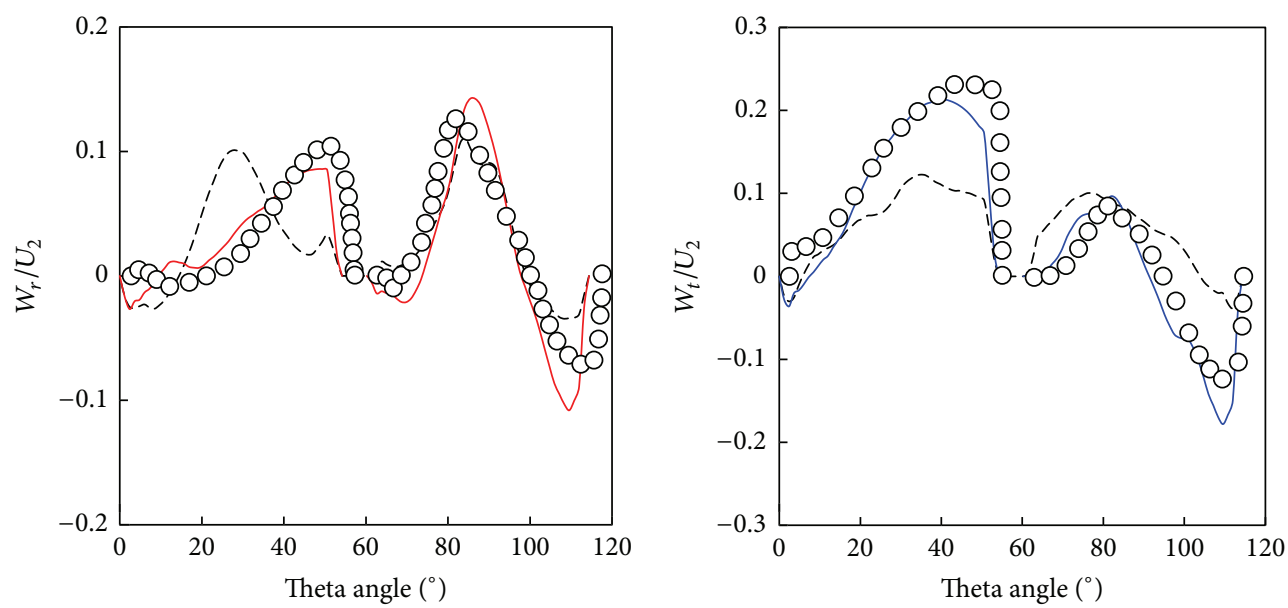

(a) Radius $R=0.50 R_{2}$
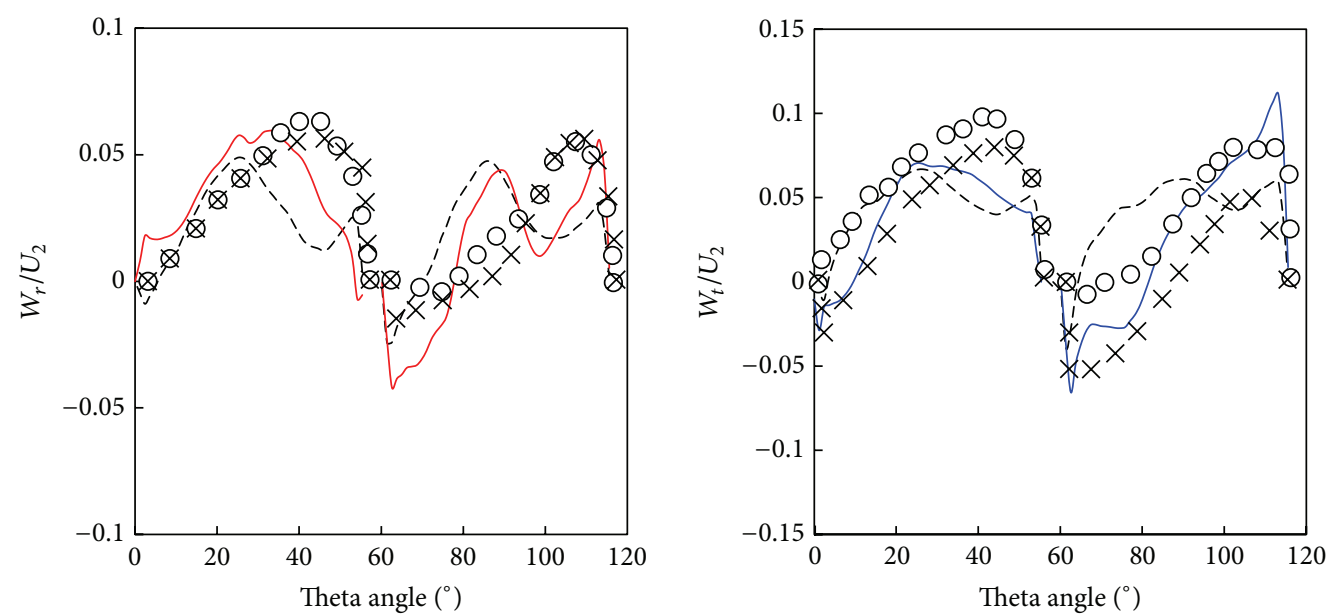

(b) Radius $R=0.75 R_{2}$
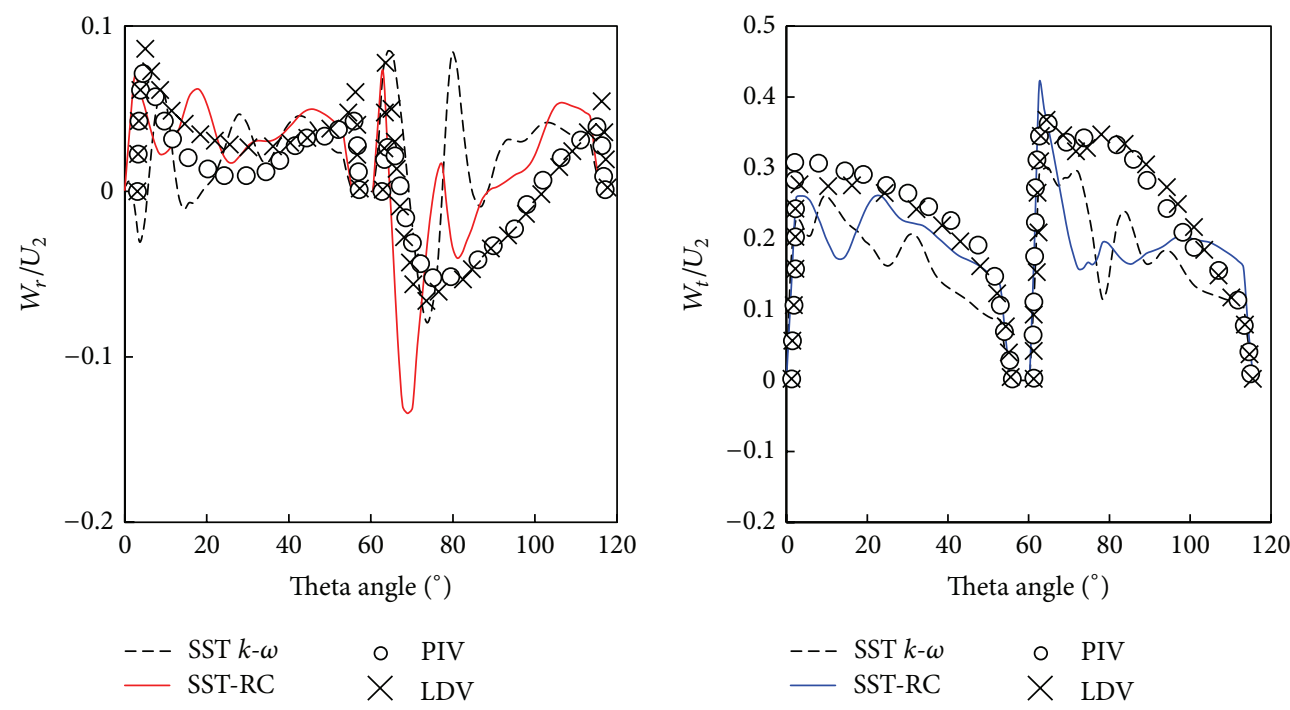

(c) Radius $R=0.98 R_{2}$

FIGURE 10: Relative velocity distributions on the spanwise $50 \%$ surface at radius of $0.50,0.75$, and $0.98 R_{2}$ under $0.25 Q_{d}$. 
pumps, if the flow regime is undesirable with strong secondary flow structures, it will be very appropriate to apply the Spalart-Shur rotation/curvature correction to the SST $k-\omega$ model and other eddy viscosity turbulence models.

\section{Conflict of Interests}

The authors declare that there is no conflict of interests regarding the publication of this paper.

\section{Acknowledgments}

The authors would like to acknowledge the financial support given by the National Natural Science Foundation of China (no. 51139007) and National "Twelfth Five-Year" Plan for Science \& Technology Support (no. 2012BAD08B03).

\section{References}

[1] J. Boussinesq, Essai sur la Théorie des Eaux Courantes, Imprimerie Nationale, Paris, France, 1877.

[2] F. G. Schmitt, "About Boussinesq's turbulent viscosity hypothesis: historical remarks and a direct evaluation of its validity," Comptes Rendus Mécanique, vol. 335, no. 9-10, pp. 617-627, 2007.

[3] P. R. Spalart and M. Shur, "On the sensitization of turbulence models to rotation and curvature," Aerospace Science and Technology, vol. 1, no. 5, pp. 297-302, 1997.

[4] Y.-N. Huang and H.-Y. Ma, "Extended intrinsic mean spin tensor for turbulence modelling in non-inertial frame of reference," Applied Mathematics and Mechanics, vol. 29, no. 11, pp. 14631475, 2008.

[5] P. Bradshaw, "The analogy between streamline curvature and buoyancy in turbulent shear flow," Journal of Fluid Mechanics, vol. 36, no. 1, pp. 177-191, 1969.

[6] A. Khodak and C. Hirsch, "Second-order non-linear k- $\varepsilon$ models with explicit effect of curvature and rotation," in Proceedings of the 3rd ECCOMAS Computational Fluid Dynamics Conference, pp. 690-696, 1996.

[7] M. L. Shur, M. K. Strelets, A. K. Travin, and P. R. Spalart, “Turbulence modeling in rotating and curved channels: assessing the Spalart-Shur correction," AIAA Journal, vol. 38, no. 5, pp. 784$792,2000$.

[8] G. Dufour, J.-B. Cazalbou, X. Carbonneau, and P. Chassaing, "Assessing rotation/curvature corrections to eddy-viscosity models in the calculations of centrifugal-compressor flows," Journal of Fluids Engineering, Transactions of the ASME, vol. 130, no. 9, 2008 .

[9] J.-B. Cazalbou, P. Chassaing, G. Dufour, and X. Carbonneau, "Two-equation modeling of turbulent rotating flows," Physics of Fluids, vol. 17, no. 5, Article ID 055110, 2005.

[10] P. E. Smirnov and F. R. Menter, "Sensitization of the SST turbulence model to rotation and curvature by applying the Spalart-Shur correction term," Journal of Turbomachinery, vol. 131, no. 4, Article ID 041010, 2009.

[11] T. P. Dhakal and D. K. Walters, "A Three-equation variant of the SST k-w model sensitized to rotation and curvature effects," Journal of Fluids Engineering, vol. 133, no. 11, Article ID 111201, 2011.
[12] W. D. York, D. K. Walters, and J. H. Leylek, "A simple and robust linear eddy-viscosity formulation for curved and rotating flows," International Journal of Numerical Methods for Heat \& Fluid Flow, vol. 19, no. 6, pp. 745-776, 2009.

[13] A. Hellsten, "Some improvements in Menter's SST k- $\omega$ turbulence model," in Proceedings of the 29th AIAA Fluid Dynamics Conference, AIAA 98-2554, Albuquerque, NM, USA, June 1998.

[14] Q. Zhang and Y. Yang, "A new simpler rotation/curvature correction method for Spalart-Allmaras turbulence model," Chinese Journal of Aeronautics, vol. 26, no. 2, pp. 326-333, 2013.

[15] C. E. Brennen, Hydrodynamics of Pumps, Cambridge University Press, Cambridge, UK, 2011.

[16] N. Pedersen, P. S. Larsen, and C. B. Jacobsen, "Flow in a centrifugal pump impeller at design and off-design conditions-part I: particle image velocimetry (PIV) and laser Doppler velocimetry (LDV) measurements," Journal of Fluids Engineering, vol. 125, no. 1, pp. 61-72, 2003.

[17] R. K. Byskov, C. B. Jacobsen, and N. Pedersen, "Flow in a centrifugal pump impeller at design and off-design conditionspart II: large eddy simulations," Journal of Fluids Engineering, Transactions of the ASME, vol. 125, no. 1, pp. 73-83, 2003.

[18] F. R. Menter, M. Kuntz, and R. Langtry, "Ten years of industrial experience with the SST turbulence model," Turbulence, Heat and Mass Transfer, vol. 4, pp. 625-632, 2003. 


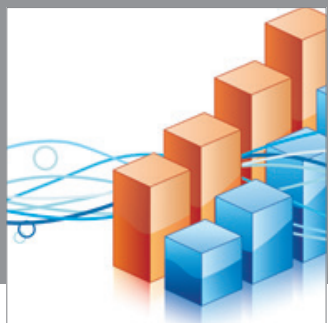

Advances in

Operations Research

mansans

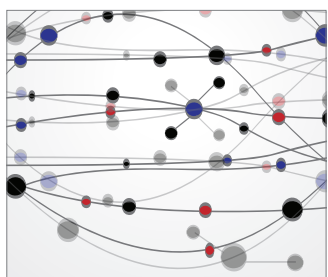

The Scientific World Journal
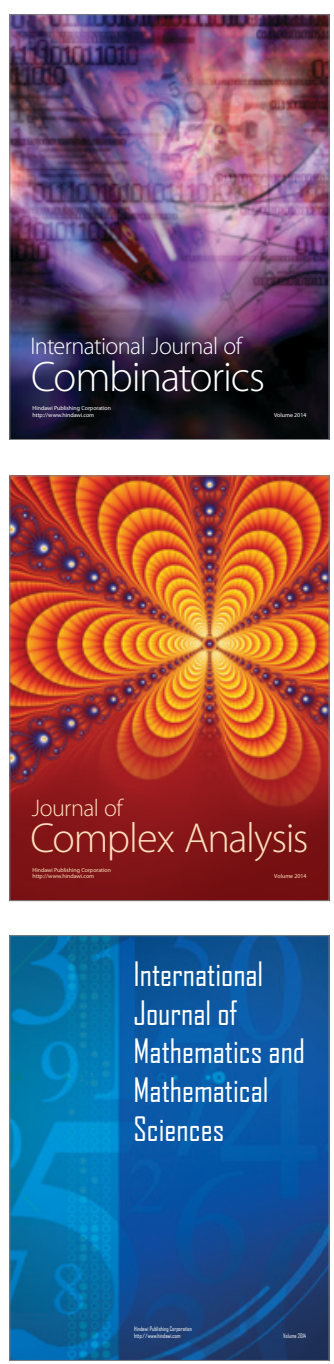
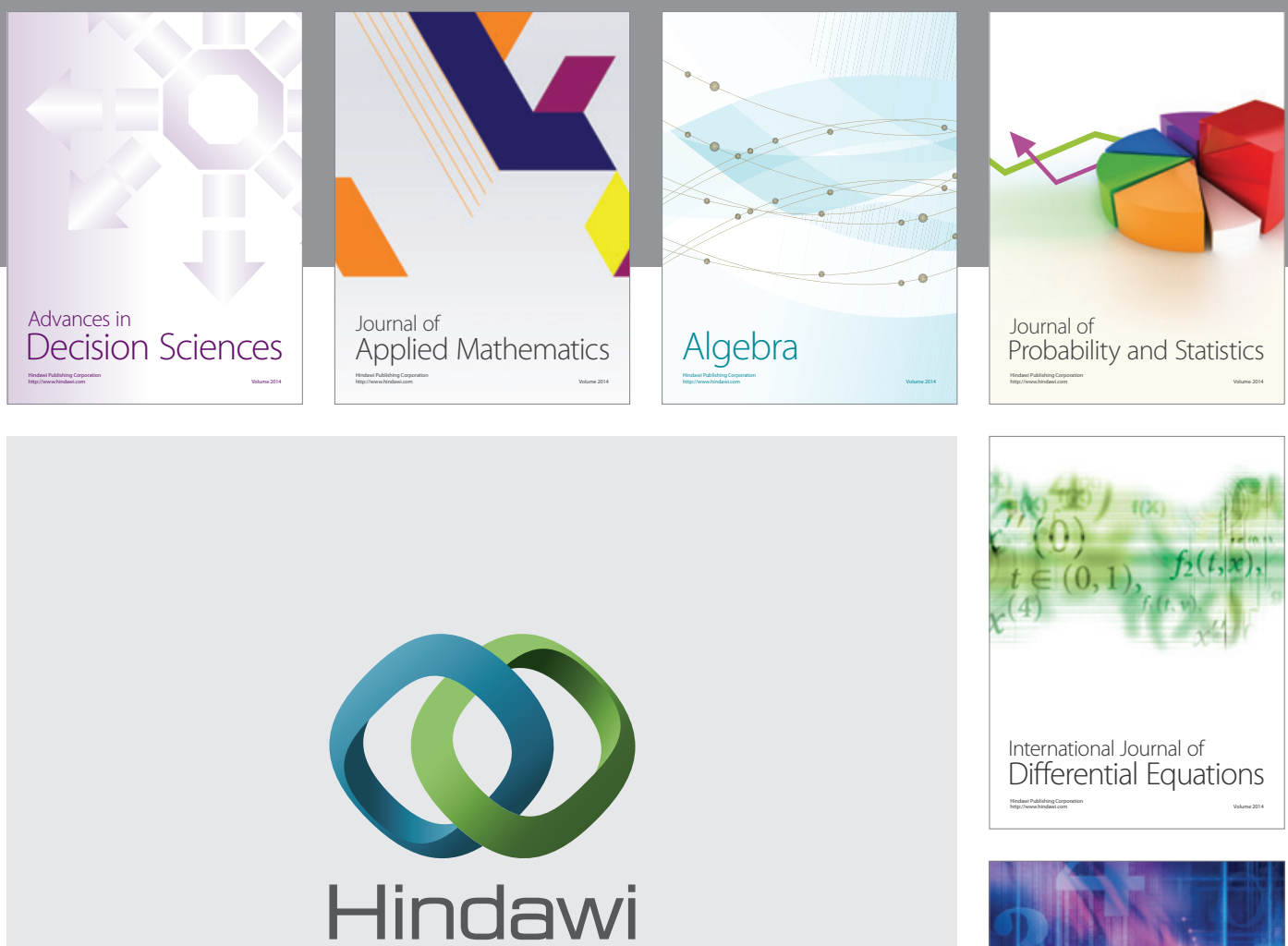

Submit your manuscripts at http://www.hindawi.com
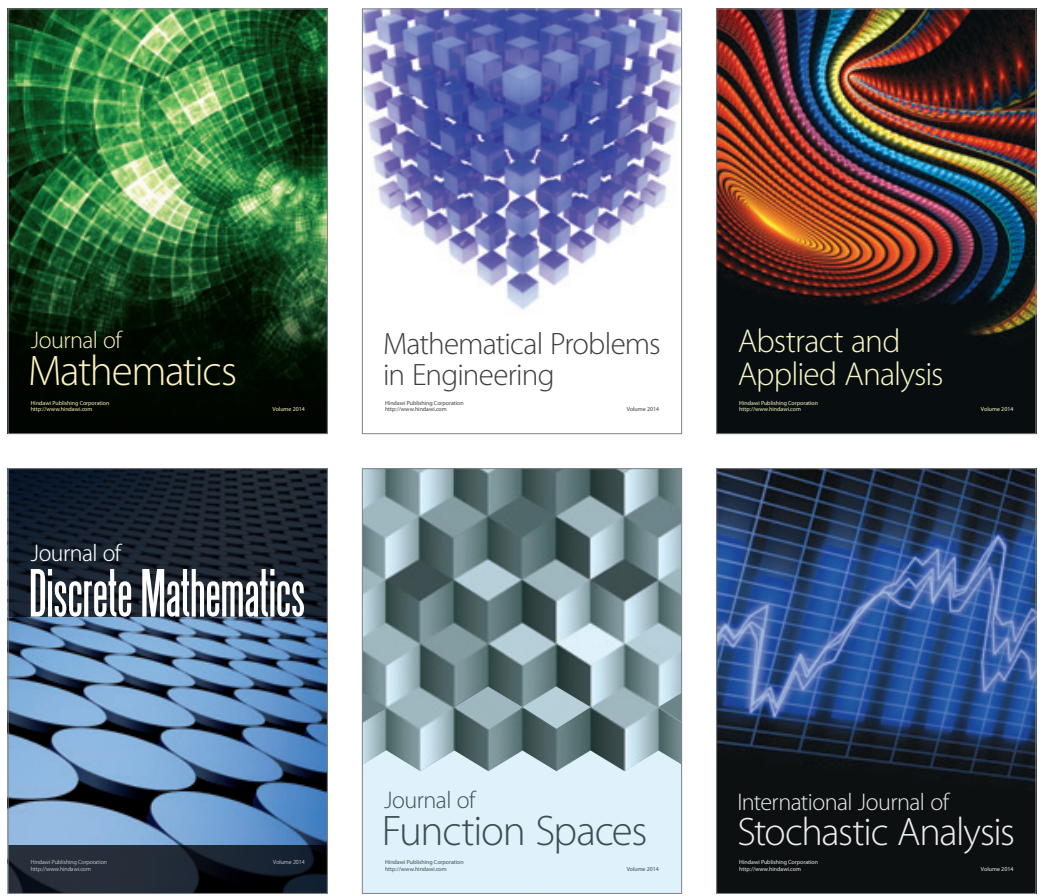

Journal of

Function Spaces

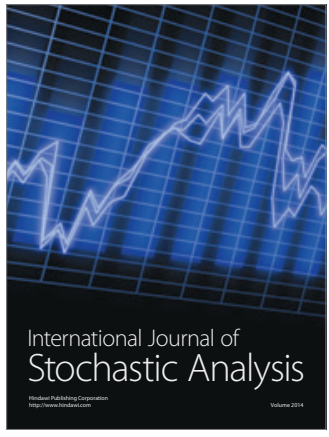

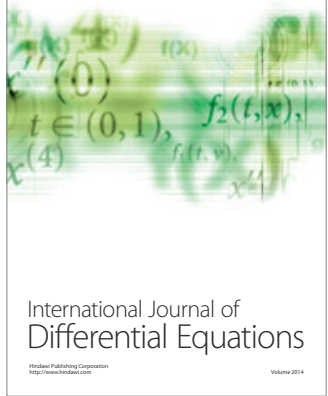
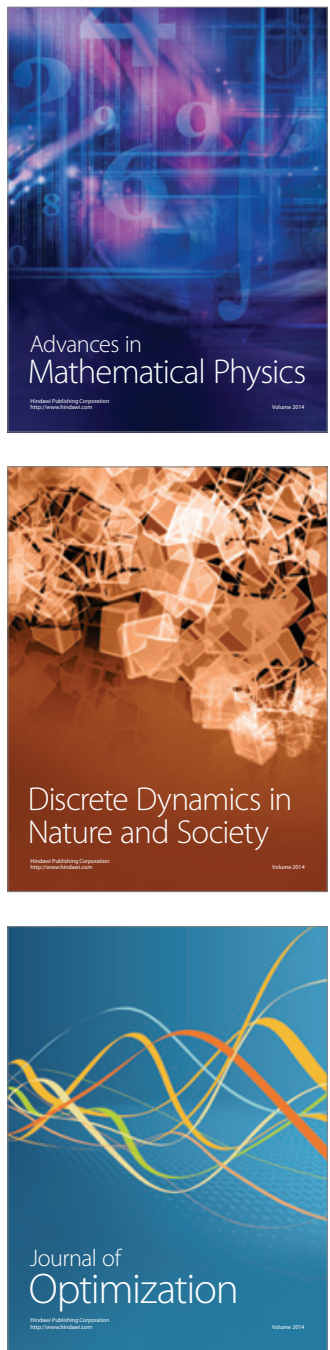